\title{
IDENTIFICATION OF STAPHYLOCOCCUS AUREUS
}

\author{
IN A FOOD POISONING INCIDENT
}

William Prince, M.D.

Gilman K. Crowell, M.S.

$\mathrm{O}^{\mathrm{N}}$ N FEBRUARY 15, 1959, a sudden, brief episode of acute gastroenteritis followed a winter carnival banquet served to approximately 180 persons representing the faculty, students, and guests of a private New Hampshire boarding school for boys in the secondary grades. Fruit cup with sherbet, rolls, roast beef with gravy, olives, celery, broccoli with hollandaise sauce, potatoes, and ice cream with fudge sauce were on the menu. Several boys in the infirmary and the school nurse also became ill after eating a meal delivered to them from the main kitchen.

On the evening of February 17, 2 days after the banquet, another outbreak occurred following a meal which included roast beef, leftover after the banquet, and baked ham as the meat dishes.

Records in the school infirmary indicated that similar incidents, although having a much lower attack rate, occurred on November 20, 1958, and on January 10, 1959.

The attack rate of the post-banquet outbreak of gastroenteritis was approximately 70 percent, with 14 percent admitted to the infirmary for treatment. All victims confined to their dormitories were supplied with medication.

Onset was characterized as sudden, with

Dr. Prince is director, bureau of communicable disease control, and Mr. Crowell is chief, bureau of food and chemistry, New Hampshire State Department of Health. severe abdominal cramps followed by nausea, vomiting, and profuse diarrhea. The incubation period varied from 2 to 4 hours. Acute symptoms subsided in most instances after 4 hours, with almost complete recovery in from 12 to 24 hours.

Physical examination revealed a low-grade fever up to $101.4^{\circ} \mathrm{F}$. in most instances. Pulse was weak and thready but improved within an hour. There was no abdominal rigidity or localized tenderness. Blood counts and urinalyses were essentially normal. Food poisoning was indicated.

\section{Investigation}

Upon questioning, it was found that the only food eaten on the night of the banquet by both the school nurse and her patients in the infirmary and the guests in the main diningroom was roast beef without gravy and broccoli without hollandaise sauce. It was also found that the students who ate leftover roast beef for the evening meal, February 17, became ill, while students who ate ham at this meal did not.

Following the banquet meal, food samples of leftover milk, hamburger which had been served at the noon meal preceding the banquet, roast beef, ice cream, chicken à la king served February 13 as the evening meal, onion soup, and chicken soup were collected in sterile containers for bacteriological study. Media used for isolation were selenite $\mathrm{F}$ broth, S.S. 
agar, desoxycholate agar, 110 medium, mannitol-salt agar, and Colbeck Ey agar. All these sampled foods were negative for Salmonella. The roast beef, however, showed a heavy growth of Staphylococcus aureus.

The school kitchen appeared tidy and well organized. Dishes were machine washed at recommended pressure and temperature. The temperature in the walk-in refrigerator was maintained between $40^{\circ}$ and $50^{\circ} \mathrm{F}$. Seven em. ployees worked in the kitchen, four men on a full-time basis and three women part time. This staff showed no visible evidence of skin lesions of infection and denied any infection or illnesses which could be related reasonably to the outbreak.

Cultures were taken from the throat and stool specimens of each member of the kitchen staff on four different occasions, at 3- to 4-week intervals. Results of these cultures showed consistently that six of the seven persons employed were positive for $S$. aureus in cultures from either or both throat and stool specimens. Cultures from stool specimens, taken from two ill students immediately following the banquet incident, were also positive.

All of the S. aureus cultures isolated from humans and food samples, when tested for coagulase reaction, were positive. Although the phage pattern of some of the cultures varied to a slight degree, all fell in the broad group 3 and were remarkably similar in their response to sensitivity tests. Throat and stool cultures of three of the seven employees in the kitchen as well as the stool cultures taken from the two ill students, exhibited an identical phage pattern $(7+/ 42 \mathrm{E} \pm / 73 \pm)$ to the culture isolated from the roast beef. The staphylococcus isolated from the roast beef, phage typed to group 3 , was found to be enterotoxigenic using the cat injection test. All cats injected with this culture filtrate exhibited vomiting within 30 minutes to 3 hours after injection.

Tests of the swabbings taken at random in the kitchen showed heavy contamination of a coagulase-positive staphylococci on the meat slicer, the meat block, and the floor. Five of the ten swabbings showed coagulase-positive staphylococci.

\section{Discussion}

It is assumed that the extensive "seeding" of the kitchen environment with staphylococci by the personnel who were carriers, coupled with unsatisfactory food handling, accounted for the repeated episodes of food poisoning at this school.

An inspection of the walk-in refrigerator showed many items of leftover food. It was apparent that the chef was not inclined to discard leftovers, preferring to keep them and serve them again at future meals.

It was also learned that it was the practice of the chef to cook all meats and other foods early in the morning of the day they were to be served, rewarming these foods just prior to serving the evening meal. Even when he was informed that this practice allowed an incubation period for food poisoning organisms, the chef was reluctant to change, since the time interval permitted the staff to enjoy an afternoon siesta.

After several educational conferences with school administrators stressing the necessity for proper handling and refrigeration of all foods, the chef was ordered to correct his practices or leave. The improper practices were corrected immediately.

The remedial measures suggested were aimed at prevention of food contamination and removal of its source from the staff and the kitchen environment. They included cleansing and sanitizing the walls, ceiling, and floors of the kitchen ; cleansing and sanitizing all kitchen utensils and work surfaces; treatment of the six kitchen staff members with antibiotics based on sensitivity studies of the cultures of staphylococcus isolated from their throats and stools; and instruction of the staff in the proper handling of food.

This study is of particular interest because of the repeated occurrence of food poisoning outbreaks traceable, not to the usual boils or other infected skin lesions of human carriers, but to pathogenic staphylococci shed from the noses, throats, and gastrointestinal tracts of chronic carriers. This type of carrier is a potential community hazard in that he may be a source of infection to others. 